\title{
Precise Control of Wavelength Channel Spacing of Microring Resonator Add-Drop Filter Array
}

Shuichi Suzuki, Yutaka Hatakeyama, Yasuo Kokubun, Member, IEEE, Member, OSA, and Sai Tak Chu, Member, IEEE

\begin{abstract}
The vertically coupled microring resonator filter is an attractive add-drop wavelength filter due to its functionality and compactness and the possibility of dense integration resulting from the cross-grid configuration. However, in the concatenated $1 \times 8$ filter array in which the ring radii are changed with the increments of $50 \mathrm{~nm}$, the wavelength channel spacing was limited to $5.7 \mathrm{~nm}$ due to the resolution limit of photomask $(50 \mathrm{~nm})$. Therefore, we developed a new technique to control precisely the channel spacing by an ultraviolet (UV)-trimming technique using polysilane as the overcladding layer. As a result, we successfully decreased the channel spacing to $0.5 \mathrm{~nm}$, and also controlled the channel spacing precisely to $1.0 \mathrm{~nm}$ using the same trimming technique.
\end{abstract}

Index Terms-Add-drop filter, microring resonator, optical waveguide device, polysilane, ultraviolet (UV) trimming.

\section{INTRODUCTION}

$\mathbf{M}$ ICRORING resonator devices are attractive as add-drop wavelength filters for wavelength multiplexed access networks, due to their functionality and compactness [1], [4]. We have proposed and demonstrated a vertically coupled microring resonator (VCMRR) filter array [5]. Fig. 1 shows the basic structure of the VCMRR filter element. Due to the stacked configuration, the upper and lower waveguides play different roles, i.e., the lower buried channel waveguides serve as input-output busline waveguides, whereas the upper ring functions as the frequency selective element. Owing to the large index difference $(\sim 34 \%)$ of the upper ring waveguide, the ring radius can be reduced to $10 \mu \mathrm{m}$, which results in a large free spectral range (FSR) of $20 \mathrm{~nm}$. In addition, due to the cross-grid topology and the compactness of filter element, a large scale integration up to $10^{4}-10^{5}$ elements $/ \mathrm{cm}^{2}$ is possible. Recently, a multichannel add-drop filter has been realized by concatenating cross-grid VCMRR elements, as shown in Fig. 2 [5]. However, the wavelength channel spacing of the add-drop filter array is realized by changing the radius of each ring is about $5.7 \mathrm{~nm}$, because the differences of ring radius are limited by the resolution of the photomask, i.e., $50 \mathrm{~nm}$. The channel spacing between the center wavelength of adjacent ports should be reduced to less than $1 \mathrm{~nm}$ for the dense wavelength division multiplexing (WDM) systems. Thus, we applied an ultraviolet

Manuscript received July 10, 2001. This work was supported in part by the Japan Society for the Promotion of Science through Grant-in-Aid for Scientific Research A 11305028, a Grant-in-Aid for Scientific Research on Priority Area A 13026210 of the Ministry of Education, Culture, Sports, Science, and Technology, by Research for the Future of Japan Society for the Promotion of Science, and by the International Communication Foundation.

S. Suzuki, Y. Hatakeyama, and Y. Kokubun are with Yokohama National University, Graduate School of Engineering, Yokohama 240-8501, Japan (e-mail: kokubun@dnj.ynu.ac.jp).

S. T. Chu is with Little Optics, Inc., Annapolis Junction, MD 20701 USA (e-mail: sai@littleoptics.com).

Publisher Item Identifier S 0733-8724(02)03342-X.

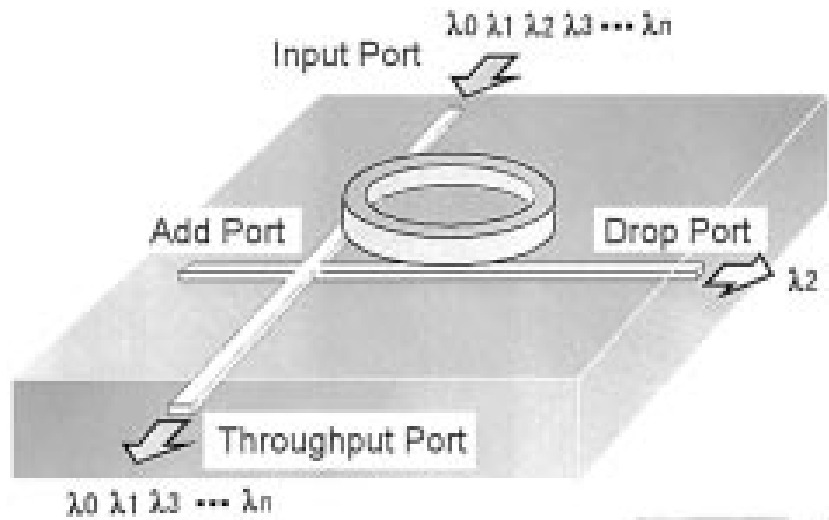

Fig. 1. Structure of VCMRR filter element.

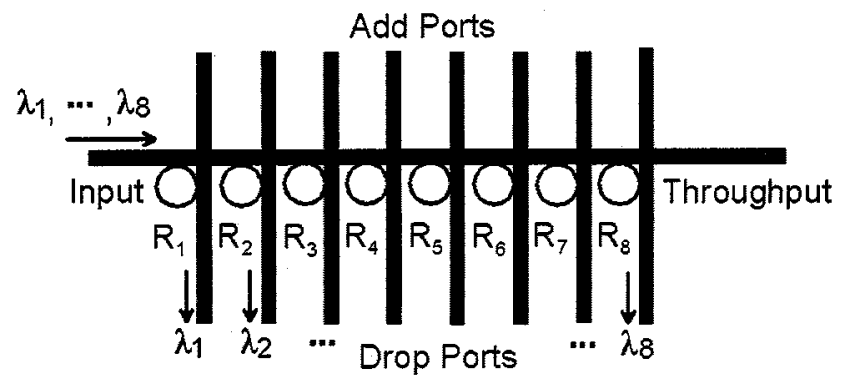

Fig. 2. VCMRR filter array.

(UV)-trimming technique [6], [7] to the multichannel add-drop filter. In this report, we describe the design of UV-trimming range and demonstrated a precise control of channel spacing of multichannel add-drop filter array using the UV-trimming technique.

\section{Design OF TRIMMING RANGE}

Fig. 3 shows the cross-sectional structure of the VCMRR filter, in which the overcladding is formed by UV-sensitive polymer. When the index of overcladding is reduced by UV irradiation, the equivalent index of the ring waveguide is also changed and the center wavelength of the filter is shifted. The polymer used in this study is polysilane $(\mathrm{MeSi})_{n}[\mathrm{Me}(\mathrm{EtO}) \mathrm{Si}]_{m}$ $(n / m$ : mixture ratio) [8], [9]. The measured refractive index change of the polysilane by UV irradiation is shown in Fig. 4. The peak wavelength of the UV light (Hg lamp) used in this experiment was $370 \mathrm{~nm}$ and the intensity was $20 \mathrm{~mW} / \mathrm{cm}^{2}$. The refractive index was decreased from 1.51 to 1.43 by the UV irradiation for $400 \mathrm{~s}$.

We calculated the amount of center wavelength sift and the bending loss of the ring waveguide when the index of overcladding was assumed to be reduced from 1.51 to 1.43 . The 


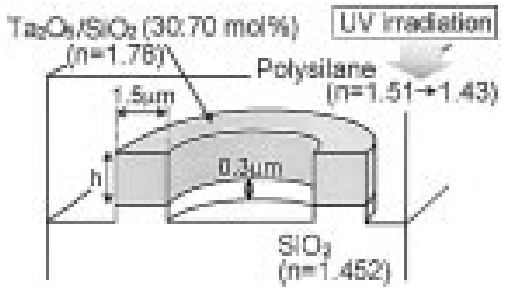

Fig. 3. Principle of UV trimming.

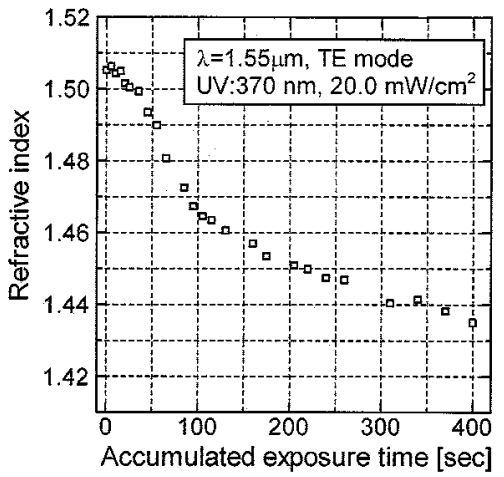

Fig. 4. Measured refractive index change of polysilane versus UV exposure time.

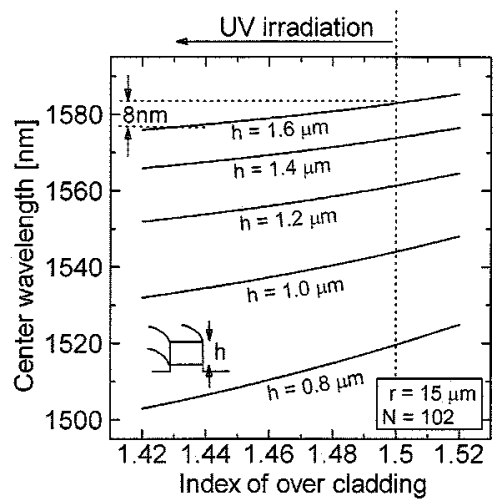

Fig. 5. Calculated center wavelength shift by trimming.

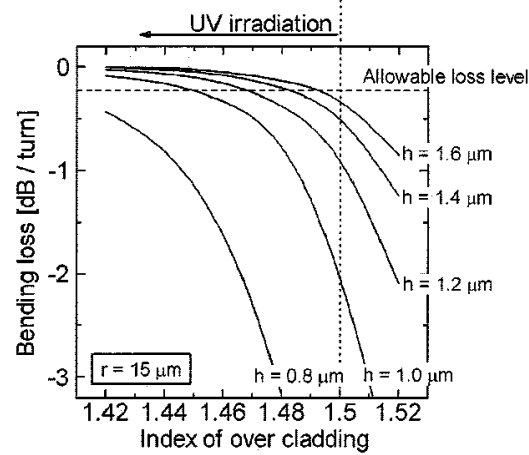

Fig. 6. Calculated radiation loss of ring vs index of overcladding.

parameters used in the calculation are given in Fig. 3. The calculated result of the wavelength shift and the bending loss for the ring radius of $15 \mu \mathrm{m}$ is shown in Figs. 5 and 6, respectively. The semivectorial finite difference method [10] was used

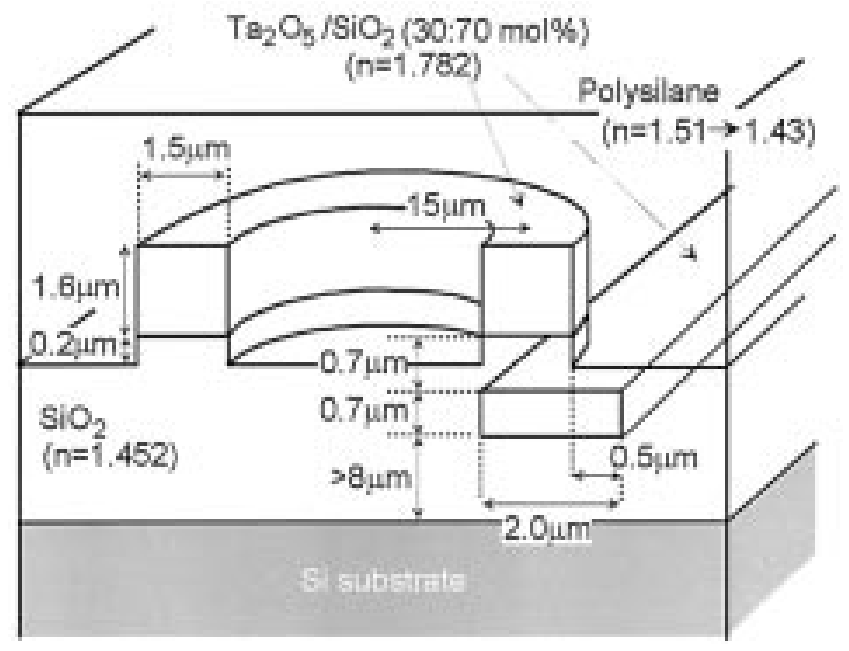

Fig. 7. Parameters of fabricated VCMRR filter.

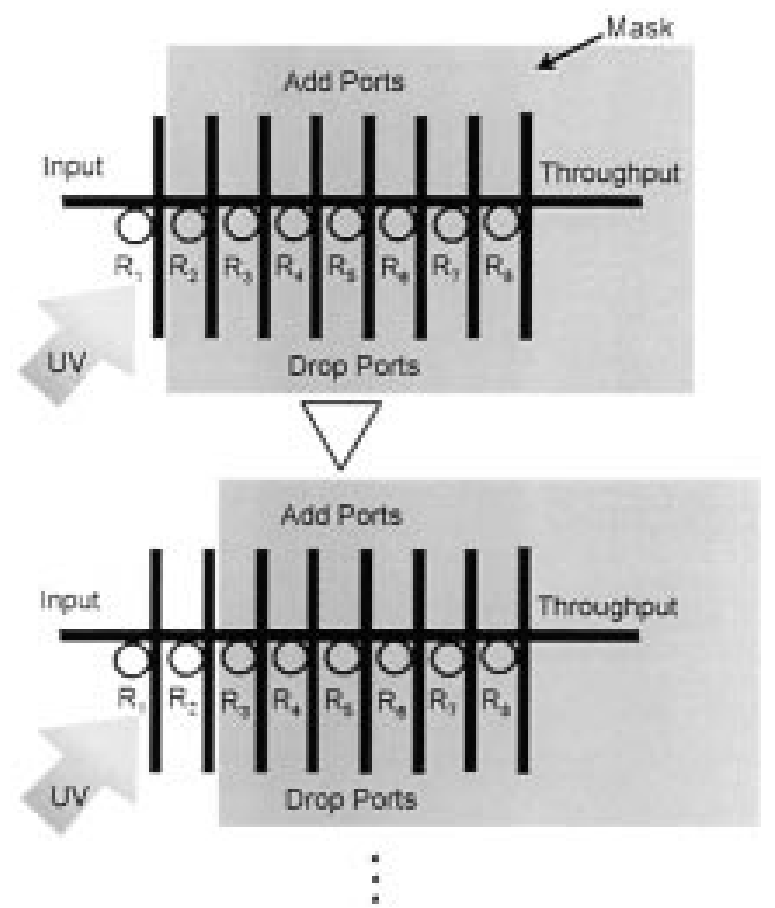

Fig. 8. Way of UV irradiation on each ring with different exposure time.

in this analysis to calculate the complex propagation constant. The center wavelength was calculated from the real part of the propagation constant, and the bending loss was calculated from the imaginary part of the propagation constant.

It can be seen that when the core thickness of ring waveguide is smaller, the range of center wavelength shift by trimming is larger. However, because the bending loss is also larger as the decrease of core thickness, we designed the thickness of the ring core to be $1.6 \mu \mathrm{m}$, so that the bending loss of the ring waveguide is the same level as the value for which we could previously obtain a clear spectrum response [11]. For this designed structure, the trimming range of $8 \mathrm{~nm}$ can be obtained, whereas the bending loss is kept smaller than the allowable loss level ( 0.3 $\mathrm{dB}$ per turn). 


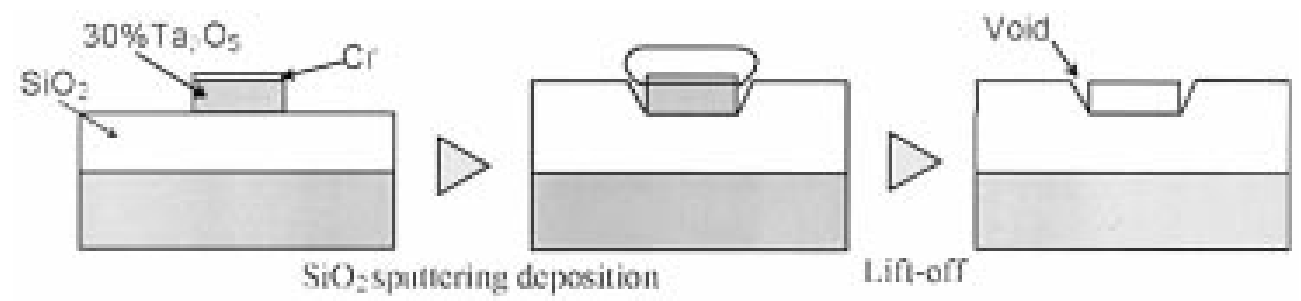

Fig. 9. Problem of conventional liftoff process.

\section{DEVICE FABRICATION}

We fabricated a $1 \times 8$ filter array, as shown in Fig. 2, in which the ring radii $(15 \mu \mathrm{m})$ and the ring core thicknesses $(1.6 \mu \mathrm{m})$ are identical for all ports. Those microrings are named ring $1-8$, respectively. The channel spacing between adjacent ports is $250 \mu \mathrm{m}$. Fig. 7 shows the cross-sectional structure of the basic element of fabricated VCMRR filter array. The substrate cladding and separation layer are made of $\mathrm{SiO}_{2}(n=1.452$ at $\lambda=1.55 \mu \mathrm{m})$. The ring and busline cores are made of the $\mathrm{Ta}_{2} \mathrm{O}_{5} / \mathrm{SiO}_{2}$ compound glass to give a refractive index of 1.782 $\left(\mathrm{Ta}_{2} \mathrm{O}_{5}\right.$ mol fraction is $\left.30 \%\right)$. The microring cores were covered by the polysilane with the thickness of $10 \mu \mathrm{m}$. Other sizes of the device are shown in Fig. 7. Each ring was irradiated by UV light with different exposure time by shifting a metal mask using a manipulator, as shown in Fig. 8.

Details of the fabrication process were reported in [12]. $\mathrm{SiO}_{2}$ and $\mathrm{Ta}_{2} \mathrm{O}_{5} / \mathrm{SiO}_{2}$ compound glass layers were deposited by a radiofrequency (RF) sputtering technique. A $\mathrm{Cr}$ mask was formed by vacuum evapolation and was patterned by photolithography. The channel waveguide was fabricated by reactive ion etching (RIE) using $\mathrm{CF}_{4}$ gas. The polysilane overcladding was dip coated. It is especially important for the stacked configuration, such as the VCMRR filter, to flatten the top surface of the lower buried waveguide layer. We have used the liftoff process shown in Fig. 9 to flatten the lower waveguide. There was a problem, however, that the voids arise at the vertical side walls of the lower core, and this causes the dent to appear on the top surface of the buried waveguide. Thus, we used a bias-sputtering technique to bury the lower waveguide core by $\mathrm{SiO}_{2}$ cladding. The comparison between the surface roughness by the previous liftoff process and that by the inproved process using a bias sputtering is shown in Fig. 10(a) and (b), which show the cross-sectional SEM images of the buried busline waveguide. It can be seen that the dent on the top surface of the lower waveguide was improved to be less than $0.1 \mu \mathrm{m}$. Fig. 11 shows the optical microscope image of the fabricated device.

\section{MEASUREMENT OF VCMRR FILTER ARRAY}

Using the improved fabrication process, we fabricated a $1 \times 8$ concatenated filter array with identical ring radius $(15 \mu \mathrm{m})$ for all microring resonators. Although eight filter elements were integrated in this device, we used rings 2 and 7 for the demonstration of the precise control of channel spacing. This is because the initial channel spacing between these two was the largest due to the thickness nonuniformity of the core layer, and was suitable for the demonstration of large amount of trimming. In the

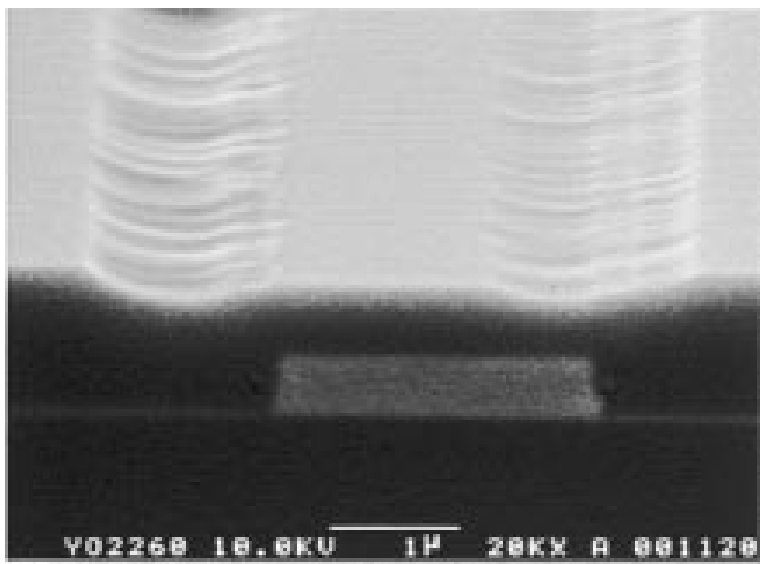

(a)

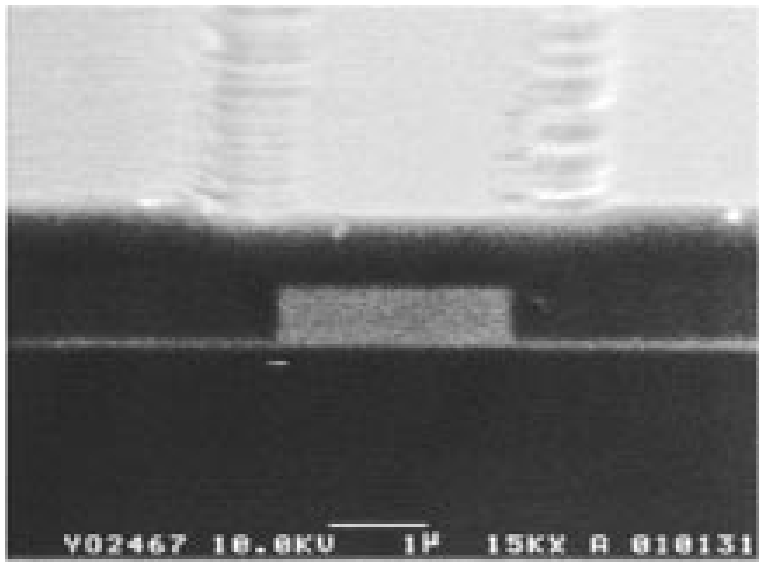

(b)

Fig. 10. Cross-sectional structure of busline waveguide. (a) Conventional liftoff. (b) Improved liftoff.

measurement of filter response, a tunable laser diode [LD (Anritsu MG9638A)] was used as the light source and the output spetrum was measured by an optical spectrum analyzer (Anritsu MS9710B, Tokyo, Japan) that was synchronously operated with the tunable laser.

Fig. 12 shows the measured spectrum responses of rings 2 and 7 in the filter array before controlling the channel spacing. After the fabrication, UV light from an $\mathrm{Hg}$ lamp was irradiated to overall filter array for $80 \mathrm{~s}$ through a bundle fiber. The exposure condition of the UV light source used in this experiment was the same as used for the measurement of index change of the polysilane film, i.e., the power density was $20 \mathrm{~mW} / \mathrm{cm}^{2}$ and the peak wavelength was $370 \mathrm{~nm}$. This irradiation was needed to obtain a clear spectrum by reducing the index of polysilane overcladding because the initial refractive index of polysilane 


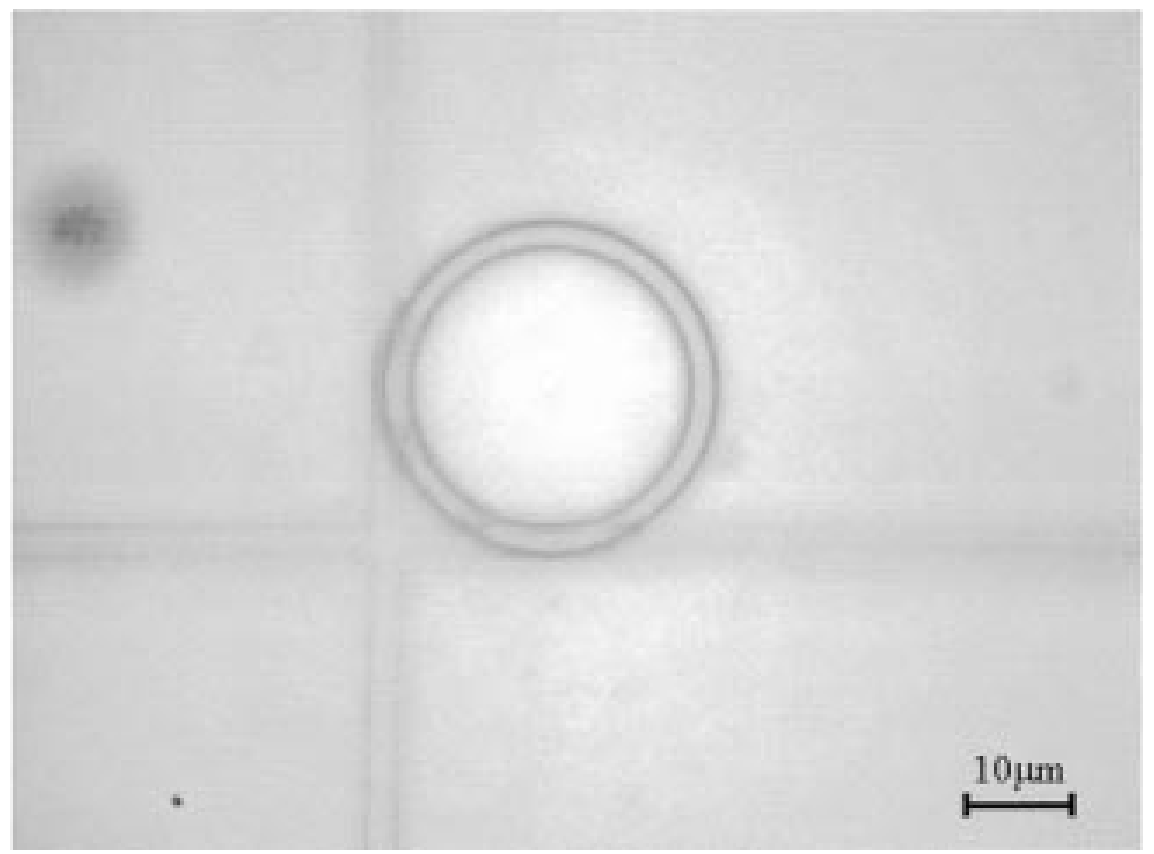

Fig. 11. Optical microscope image of fabricated ring resonator.

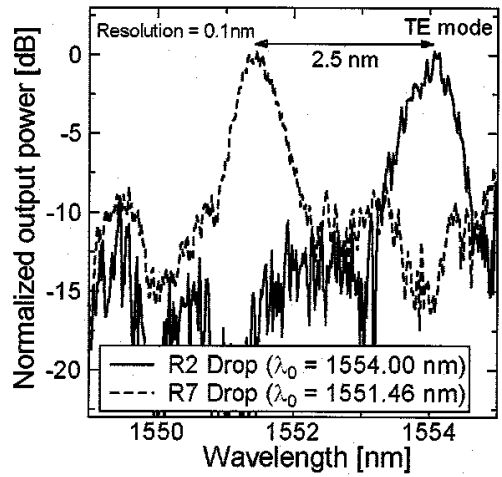

Fig. 12. Measured spectrum responses of rings 2 and 7 before channel spacing control.

(1.51) is a little larger than the value required to guarantee the low-loss propagation in the ring. Therefore, to improve the confinement in the ring waveguide and to reduce the radiation loss of the ring waveguide, the preexposure of UV light was needed. The channel spacing between rings 2 and 7 shown in Fig. 12 is about $2.5 \mathrm{~nm}$. To reduce the spacing, we irradiated the UV light only on ring 2 which has a longer center wavelength than that of ring 7. The spectrum responses after 140-s irradiation on ring 2 are shown in Fig. 13. Comparing Fig. 12 with Fig. 13, it can be seen that the channel spacing between rings 2 and 7 was successfully reduced from 2.5 to $0.5 \mathrm{~nm}$.

Fig. 14 shows the change of the spectrum response of ring 2 for different irradiation time. The center wavelength was shifted over $9.7 \mathrm{~nm}$ by 220 -s irradiation. Fig. 15 is the spectrum responses after $500 \mathrm{~h}$ and shows the spectrum change due to the aging. The center wavelengths of rings 2 and 7 changed from those in Fig. 13 by 0.07 and $0.31 \mathrm{~nm}$, respectively, and the channel spacing was increased to $0.7 \mathrm{~nm}$. The center wavelength change seems to be caused by the index change of polysilane

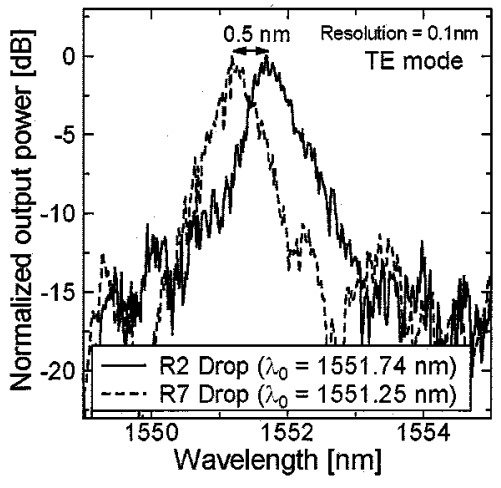

Fig. 13. Measured spectrum responses of rings 2 and 7 after UV irradiation on ring 2 .

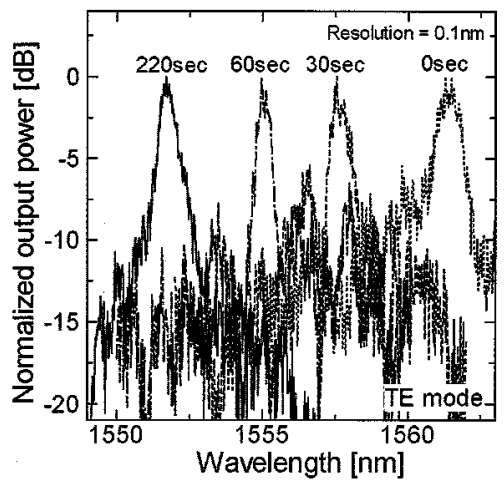

Fig. 14. Change of spectrum response of ring 2 by UV irradiation.

overcadding due to the effect of the oxidation or humidity absorption. The difference of center wavelength change between rings 2 and 7 can be attributed to the difference of UV irradiation time. Because ring 2 was irradiated for more time $(220 \mathrm{~s})$ than 7 $(80 \mathrm{~s})$, the index change of polysilane was nearly saturated and 


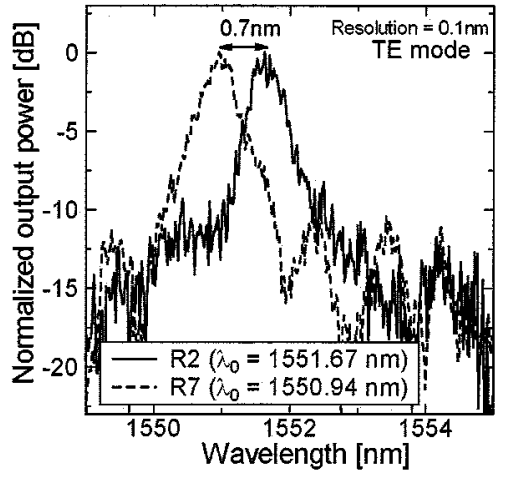

Fig. 15. Change of spectrum response due to 500-h aging.

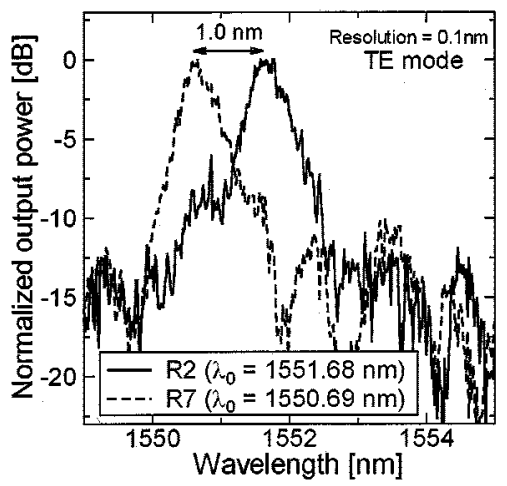

Fig. 16. Measured spectrum responses of rings 2 and 7 after UV irradiation on ring 7 .

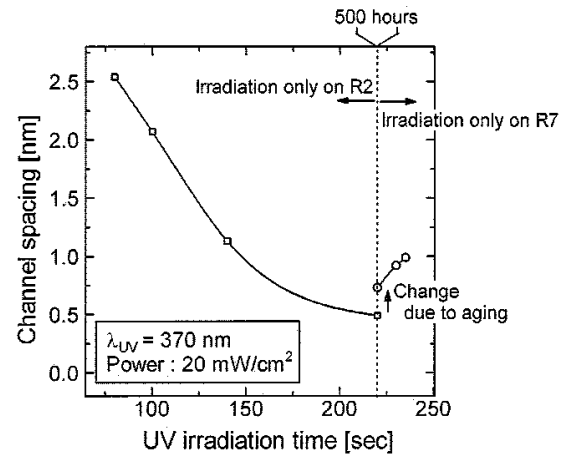

Fig. 17. Change of channel spacing between rings 2 and 7 by UV irradiation.

the aging effect was smaller than that of ring 7 . To prevent this aging effect, a hermetic packaging will be required for practical use.

Fig. 16 shows the spectrum responses when only ring 7 was exposed to UV light for $15 \mathrm{~s}$ and the channel spacing was increased to $1.0 \mathrm{~nm}$ from $0.7 \mathrm{~nm}$. The change of channel spacing between rings 2 and 7 by UV irradiation is summarized in Fig. 17. First, both rings 2 and 7 were irradiated for $80 \mathrm{~s}$. Next, only ring 2 was irradiated for $140 \mathrm{~s}$, and the channel spacing was decreased to $0.5 \mathrm{~nm}$. The vertical broken line means the time interval of $500 \mathrm{~h}$, and the channel spacing was slightly increased to $0.7 \mathrm{~nm}$. For the last $15 \mathrm{~s}$, only ring 7 was exposed to the UV light and the channel spacing was increased to $1.0 \mathrm{~nm}$.

\section{CONCLUSION}

We demonstrated the precise control of channel spacing of vertically coupled microring resonator add-drop filter array by using a UV-trimming technique. In the design, we found the optimum structure which satisfies both the wide trimming range $(\sim 8 \mathrm{~nm})$ and the low bending loss of the ring waveguide $(\leq 0.3 \mathrm{~dB} /$ turn $)$. In the fabrication, we improved the liftoff process and reduced the dent on the top of the lower waveguide. By the step-by-step UV irradiation on the ring resonator in the filter array, a narrow channel spacing of $0.5 \mathrm{~nm}$ was successfully obtained and the channel spacing was inversely increased to $1.0 \mathrm{~nm}$ by exchanging the filter under exposure. Thus, we successfully demonstrated the precise control of center wavelength and the channel spacing.

\section{ACKNOWLEDGMENT}

The authors wish to thank Dr. T. Imai, Dr. T. Fujimoto, and Dr. K. Ando of GE Toshiba Silicones Company, Ltd., for supplying polysilane.

\section{REFERENCES}

[1] B. E. Little, S. T. Chu, H. A. Haus, J. Foresi, and J. P. Laine, "Microring resonator channel dropping filters," J. Lightwave Technol., vol. 15, pp. 998-1005, June 1997.

[2] C. K. Madsen, G. Lents, A. J. Bruce, M. A. Capuzzo, L. T. Gomez, T. N. Nielsen, and I. Brener, "Multistage dispersion compensator using ring resonators," Opt. Lett., vol. 24, no. 22, pp. 1555-1557, 1999.

[3] R. Grover, P. P. Absil, V. Van, J. V. Hryniewicz, B. E. Little, O. King, L. C. Calhourn, F. G. Johnson, and P.-T. Ho, "Vertically coupled GaInAsP-InP microring resonators," Opt. Lett., vol. 26, no. 8, pp. 506-508, 2001.

[4] J. V. Hryniewicz, P. P. Absil, B. E. Little, R. A. Wilson, and P.-T. Ho, "Higher order filter response in coupled microring resonators," IEEE Photon. Technol. Lett., vol. 12, pp. 506-508, Nov. 2000.

[5] S. T. Chu, B. E. Little, W. Pan, T. Kaneko, S. Sato, and Y. Kokubun, "An eight-channel add-drop filter using vertically coupled microring resonators over a cross grid," IEEE Photon. Technol. Lett., vol. 11, pp. 691-693, June 1999.

[6] S. Sato, W. Pan, S. T. Chu, S. Endo, S. Suzuki, and Y. Kokubun, "59-nm trimming of center wavelenth of ARROW-type vertical coupler filter by UV irradiakon," IEEE Photon. Technol. Lett., vol. 11, pp. 358-360, Mar. 1999.

[7] S. T. Chu, W. Pan, S. Sato, T. Kaneko, B. E. Little, and Y. Kokubun, "Wavelength trimming of a microring resonator filter by means of a UV sensitive polymer overlay," IEEE Photon. Technol. Lett., vol. 11, pp. 688-690, June 1999.

[8] K. Kabeta, S. Wakamatsu, and T. Imai, "Preparation of substituted network polysilanes by disproportionation reaction of alkoxydisilanes in the presence of alkoxysilanes," J. Polymer Sci. Part A: Polymer Chem., vol. 34, pp. 2991-2998, 1996.

[9] K. Kabeta, S. Sugi, S. Wakamatsu, and T. Imai, "Photoreaction of alkoxypolysilanes," Polymer, vol. 38, no. 20, pp. 5015-5021, 1997.

[10] W. P. Huang, C. L. Xu, W. Lui, and K. Yokoyama, "The perfectly matched layer boundary condition for modal analysis of optical waveguides," IEEE Photon. Tech. Lett., vol. 8, pp. 649-651, May 1996.

[11] S. T. Chu, W. Pan, S. Suzuki, B. E. Little, S. Sato, and Y. Kokubun, "Temperature insensitive vertically coupled microring resonator Add-Drop filters by means of a polymer overlay," IEEE Photon. Technol. Lett., vol. 11, pp. 1138-1140, Sept. 1999.

[12] B. E. Little, S. T. Chu, W. Pan, D. Ripin, T. Kaneko, Y. Kokubun, and E. Ippen, "Vertically coupled glass microring resonator channel dropping filters," IEEE Photon. Technol. Lett., vol. 11, pp. 215-217, Feb. 1999. 


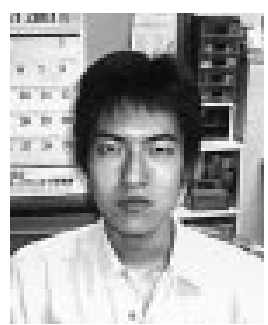

(IEICE) of Japan.

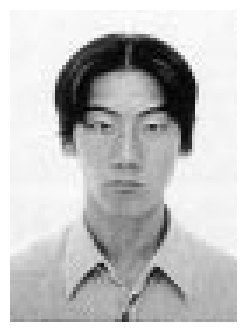

Yutaka Hatakeyama was born in Tokyo, Japan, on Februaly 20, 1979. He received the B.E. degree in electrical and computer engineering from Yokohama National University, Yokohama, Japan, in 2001. He is currently working toward the M.E. degree at Yokohama National University.

His research interests include optical waveguide-type functional devices.

Mr. Hatakeyama is a member of the Japan Society of Applied Physics (JSAP).

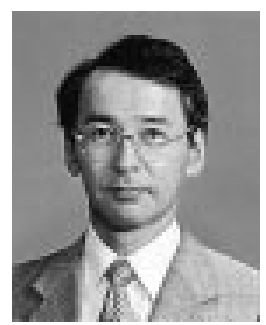

Yasuo Kokubun (M'85) was born in Fukushima, Japan, on July 7, 1952. He received the B.E. degree from Yokohama National University, Yokohama, Japan, in 1975 and the M.E. and Dr. Eng. degrees from Tokyo Institute of Technology, Tokyo, Japan, in 1977 and 1980, respectively.

From 1980 to 1983, he was with the Research Laboratory of Precision Machinery and Electronics, Tokyo Institute of Technology, as a Research Associate. In 1983, he joined Yokohama National University as an Associate Professor, and is now a Professor of the Department of Electrical and Computer Engineering. His current research is in integrated photonics, especially waveguide-type functional devices and three-dimentional integrated photonics. From 1984 to 1985, he was with AT\&T Bell Laboratories, Holmdel, NJ, as a Visiting Reseacher and was engaged in a novel waveguide on semiconductor substrate (ARROW) for integrated optics. From 1996 to 1999, he served as the Project Leader of the three-dimensional microphotonics project at the Kanagawa Academy of Science and Technology, Kanagawa, Japan.

Prof. Kokubun is a member of the Optical Society of America (OSA) and the Japan Society of Applied Physics (JSAP).

Sai Tak Chu (M'90), photograph and biography not available at the time of publication. 